\title{
Abelian varieties over finite fields with a specified characteristic polynomial modulo $\ell$
}

\author{
par JOSHUA HOLDEN
}

\begin{abstract}
RÉSUMÉ. Nous estimons la fraction des classes d'isogénie des variétés abeliennes sur un corps fini qui possèdent un polynôme caractéristique donné $P(T)$ modulo $\ell$. Comme application nous trouvons la proportion des classes d'isogénie des variétés abeliennes qui possèdent un point rationnel d'ordre $\ell$.
\end{abstract}

ABSTRACT. We estimate the fraction of isogeny classes of abelian varieties over a finite field which have a given characteristic polynomial $P(T)$ modulo $\ell$. As an application we find the proportion of isogeny classes of abelian varieties with a rational point of order $\ell$.

\section{Introduction}

Let $\mathbf{F}$ be a finite field of characteristic $p$ and order $q$, and $\ell$ a prime not equal to $p$. Let

$$
\begin{aligned}
& P(T)= \\
& \quad\left(T^{2 g}+q^{g}\right)+a_{1}\left(T^{2 g-1}+q^{g-1} T\right)+\cdots+a_{g-1}\left(T^{g+1}+q T^{g-1}\right)+a_{g} T^{g}
\end{aligned}
$$

be a polynomial. The goal of this paper is to estimate the number of isogeny classes of abelian varieties over $\mathbf{F}$ of dimension $g$ for which the characteristic polynomial for the action of Frobenius is congruent to $P(T)$ modulo $\ell$.

The initial motivation for this problem came from the following question, posed in [4] and related to the Fontaine-Mazur Conjecture for number fields: Question 1. Let $k$ be a function field over a finite field $\mathbf{F}$ of characteristic $p$ and order $q$, and $\ell$ a prime not equal to $p$. Let $K=k \mathbf{F}_{\ell \infty}$ be obtained from $k$ by taking the maximal $\ell$-extension of the constant field. If $M$ is an unramified $\ell$-adic analytic $\ell$-extension of $k$, and $M$ does not contain $K$, must $M$ be a finite extension of $k$ ?

In general the answer to Question 1 is no, with examples due to Ihara ([5]) and to Frey, Kani, and Völklein ([3]). However, the following theorems were proved in [4]:

Manuscrit reçu le 5 septembre 2002. 
Theorem 1 (Theorem 2 of [4]). Let $k_{0}$ be a function field over a finite field of characteristic $p$, and let $k$ be a constant field extension. Let $\ell$ be a prime not equal to $p$. If $\ell$ does not divide the class number $P(1)$ of $k_{0}$, then any everywhere unramified powerful (a fortiori uniform) pro- $\ell$ extension of $k$, Galois over $k_{0}$, with no constant field extension, is finite.

Theorem 2 (See Corollary 4.11 of [4]). Let $k_{0}$ be a function field over a finite field of characteristic $p$, and let $k$ be a constant field extension. Let $\ell$ be a prime not equal to $p$. Let $P(T)$ be the characteristic polynomial of Frobenius for the Jacobian of the curve associated with $k_{0}$. Suppose that the distinct roots of $P(T)$ modulo $\ell$ (possibly in some extension of $\mathbf{Z} / \ell \mathbf{Z}$ ) consist of $\lambda_{0}, \lambda_{1}, \ldots, \lambda_{n}$ such that for all $i \neq j, \lambda_{i} \lambda_{j} \neq 1$. Suppose further that if any $\lambda_{i}=1, \lambda_{i}$ is at most a double root of $P(T)$ modulo $\ell$, and if any $\lambda_{i}=-1, \lambda_{i}$ is only a simple root of $P(T)$ modulo $\ell$. Then there are no unramified infinite powerful pro- $\ell$ extensions of $k_{n}$, Galois over $k_{0}$, with no constant field extension.

In the paper [1], Jeffrey Achter and the author address the question of how many function fields are associated with a given $P(T)$ modulo $\ell$, and thus how many fall under the purview of Theorem 1 and Theorem 2. In this paper we will address the different but related question of how many isogeny classes of abelian varieties have a given characteristic polynomial $P(T)$ modulo $\ell$. As an application we find the proportion of isogeny classes of abelian varieties with a rational point of order $\ell$.

We have chosen the following way to address these questions, starting with the application to rational points. Fix distinct primes $p$ and $\ell$. For each $r$, let $\mathbf{F}_{p^{r}}$ be the finite field with $p^{r}$ elements. By the work of Tate and Honda, two abelian varieties are isogenous if and only if they have the same zeta function. Thus to each isogeny class of abelian varieties defined over $\mathbf{F}_{p^{r}}$ we associate the unique polynomial $P(T)$ (the Weil polynomial or Weil q-polynomial) which is the characteristic polynomial for the action of Frobenius and the reciprocal of the numerator of the zeta function of any variety in the isogeny class. Then $\ell$ divides $P(1)$ if and only if each abelian

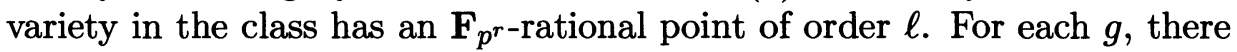
are finitely many isogeny classes of abelian varieties with dimension $g$. Let $d_{r, g}$ be the fraction of isogeny classes of dimension $g$ over $\mathbf{F}_{p^{r}}$ for which $\ell$ divides $P(1)$. Then

Theorem 3. For fixed $g$,

$$
\lim _{r \rightarrow \infty} d_{r, g}=\frac{1}{\ell} .
$$

This result and the other major result of this paper could also be obtained using the techniques of [1]. The proofs given here are perhaps more elementary, and also give some access to the number of isogeny classes and not merely the proportion satisfying each condition. 


\section{Lattices}

The proof of Theorem 3 relies on the method of counting abelian varieties introduced by DiPippo and Howe in [2]. Let $q=p^{r}$ and $I(q, g)$ be the number of isogeny classes of $g$-dimensional abelian varieties over $\mathbf{F}_{q}$. Let $P(T)$ be as before. If $P(T)$ is associated to the isogeny class of a $g$-dimensional abelian variety then $P(T)$ has degree $2 g$. Write

$$
P(T)=\prod_{j=1}^{2 g}\left(T-\alpha_{j}\right) .
$$

Then $P(T)$ has the property that $\left|\alpha_{j}\right|=q^{1 / 2}$, and the real roots, if any, have even multiplicity. Note that since the possible Weil polynomials $P(T)$ for a given $g$ are monic integral polynomials of fixed degree and have roots (and therefore coefficients) of bounded size, there are only finitely many of them. Thus $I(q, g)$ is finite.

If we write

$$
\begin{aligned}
& P(T)= \\
& \quad\left(T^{2 g}+q^{g}\right)+a_{1}\left(T^{2 g-1}+q^{g-1} T\right)+\cdots+a_{g-1}\left(T^{g+1}+q T^{g-1}\right)+a_{g} T^{g}
\end{aligned}
$$

and let $Q(T)=P\left(q^{1 / 2} T\right) / q^{g}$, then $P(T)$ is associated with another polynomial

$$
Q(T)=\left(T^{2 g}+1\right)+b_{1}\left(T^{2 g-1}+T\right)+\cdots+b_{g-1}\left(T^{g+1}+T^{g-1}\right)+b_{g} T^{g} .
$$

Let $V_{g}$ be the set of vectors $\mathbf{b}=\left(b_{1}, \ldots, b_{g}\right)$ in $\mathbf{R}^{g}$ such that all of the complex roots of $Q(T)$ lie on the unit circle and all real roots occur with even multiplicity. Let $\mathbf{e}_{1}, \ldots, \mathbf{e}_{g}$ be the standard basis vectors of $\mathbf{R}^{g}$ and let $\Lambda_{q}$ be the lattice generated by the vectors $q^{-i / 2} \mathbf{e}_{i}$. DiPippo and Howe explain that if $P(T)$ is the Weil polynomial of an isogeny class then the coefficients $a_{i}$ are such that $\left(a_{1} q^{-1 / 2}, \ldots, a_{g} q^{-g / 2}\right) \in \Lambda_{q} \cap V_{g}$. Further, let $\Lambda_{q}^{\prime}$ be the lattice generated by the vectors $q^{-1 / 2} \mathbf{e}_{1}, \ldots, q^{-(g-1) / 2} \mathbf{e}_{g-1}$ and $p q^{-g / 2} \mathbf{e}_{g}$. Then all of the polynomials $P(T)$ with coefficients $a_{i}$ such that $\left(a_{1} q^{-1 / 2}, \ldots, a_{g} q^{-g / 2}\right) \in\left(\Lambda_{q} \backslash \Lambda_{q}^{\prime}\right) \cap V_{g}$ are exactly the Weil polynomials of isogeny classes of ordinary varieties. Finally, let $\Lambda_{q}^{\prime \prime}$ be the lattice generated by the vectors $q^{-1 / 2} \mathbf{e}_{1}, \ldots, q^{-(g-1) / 2} \mathbf{e}_{g-1}$ and $s q^{-g / 2} \mathbf{e}_{g}$, where $s$ is the smallest power of $p$ such that $q$ divides $s^{2}$. Then the set of polynomials $P(T)$ with coefficients $a_{i}$ such that $\left(a_{1} q^{-1 / 2}, \ldots, a_{g} q^{-g / 2}\right) \in \Lambda_{q}^{\prime \prime} \cap V_{g}$ contains (perhaps properly) the set of Weil polynomials of isogeny classes of non-ordinary varieties.

These facts are relevant because of Proposition 2.3.1 of [2]. In a slightly generalized form, the proposition says: 
Proposition 2.1 (see 2.3.1 of [2]). Let $n>0$ be an integer and let $\Lambda \subseteq \mathbf{R}^{n}$ be a rectilinear lattice (possibly shifted) with mesh d at most $D$. Then we have

$$
\left|\#\left(\Lambda \cap V_{g}\right)-\frac{\text { volume } V_{n}}{\text { covolume } \Lambda}\right| \leq c(n, D) \frac{d}{\text { covolume } \Lambda}
$$

for some constant $c(n, D)$ depending only on $n$ and $D$ which can be explicitly computed. (We will not need the explicit computation in this paper.)

Let $v_{n}$ be the volume of $V_{n}$; Proposition 2.2.1 of [2] calculates it explicitly but we will not need that here. Let $r(q)=1-1 / p$. The lattice $\Lambda_{q}$ has covolume $q^{-g(g+1) / 4}$ and mesh $q^{-1 / 2}$. The lattice $\Lambda_{q}^{\prime}$ has covolume $p q^{-g(g+1) / 4}$, and it has mesh $q^{-1 / 2}$ unless $g=2$ and $q=p$, in which case it has mesh 1. Lastly, the lattice $\Lambda_{q}^{\prime \prime}$ has covolume $s q^{-g(g+1) / 4}$ and its mesh is at most 1 . It is then an easy consequence of the proposition that

$$
\begin{aligned}
v_{g} r(q) q^{g(g+1) / 4}-2 c(g, 1) & q^{g(g+1) / 4-1 / 2} \\
& \leq I(q, g) \\
& \leq v_{g} r(q) q^{g(g+1) / 4}+\left(v_{g}+3 c(g, 1)\right) q^{g(g+1) / 4-1 / 2} .
\end{aligned}
$$

(See [2] for details.)

Now let $I_{\ell}(q, g)$ be the number of isogeny classes of $g$-dimensional abelian varieties over $\mathbf{F}_{q}$ such that $\ell$ divides $P(1)$. Using the above notation we have

$$
P(1)=\left(1+q^{g}\right)+a_{1}\left(1+q^{g-1}\right)+\cdots+a_{g-1}(1+q)+a_{g} .
$$

Then

$$
I_{\ell}(q, g)=\sum_{\left(1+q^{g}\right)+m_{1}\left(1+q^{g-1}\right)+\cdots+m_{g-1}(1+q)+m_{g} \equiv 0} I_{(\bmod \ell)} I_{m_{1}, \ldots, m_{g}}(q, g)
$$

where $I_{m_{1}, \ldots, m_{g}}(q, g)$ is the number of isogeny classes of $g$-dimensional abelian varieties over $\mathbf{F}_{q}$ such that $a_{i} \equiv m_{i}$ modulo $\ell$. There are exactly $\ell^{g-1}$ terms on the right hand side of this expression.

Now let $\Lambda_{m_{1}, \ldots, m_{g}}$ be the lattice generated by the vectors $\ell q^{-i / 2} \mathbf{e}_{i}$ and then shifted by $\sum_{i} m_{i} q^{-i / 2} \mathbf{e}_{i}$, and let $\Lambda_{m_{1}, \ldots, m_{g}}^{\prime}=\Lambda_{m_{1}, \ldots, m_{g}} \cap \Lambda_{q}^{\prime}$ and $\Lambda_{m_{1}, \ldots, m_{g}}^{\prime \prime}=\Lambda_{m_{1}, \ldots, m_{g}} \cap \Lambda_{q}^{\prime \prime}$. Then $\Lambda_{m_{1}, \ldots, m_{g}}$ has covolume $\ell^{g} q^{-g(g+1) / 4}$ and mesh $\ell q^{-1 / 2} ; \Lambda_{m_{1}, \ldots, m_{g}}^{\prime}$ has covolume $\ell^{g} p q^{-g(g+1) / 4}$, and it has mesh $\ell q^{-1 / 2}$ unless $g=2$ and $q=p$, in which case it has mesh $\ell$; and $\Lambda_{m_{1}, \ldots, m_{g}}^{\prime \prime}$ has covolume $\ell^{g} s q^{-g(g+1) / 4}$ and mesh at most $\ell$.

We can then prove: 


\section{Proposition 2.2.}

$$
\begin{aligned}
v_{g} r(q) q^{g(g+1) / 4} \ell^{-g} & -2 c(g, \ell) q^{g(g+1) / 4-1 / 2} \ell^{1-g} \\
& \leq I_{m_{1}, \ldots, m_{g}}(q, g) \\
& \leq v_{g} r(q) q^{g(g+1) / 4} \ell^{-g}+\left(v_{g}+3 c(g, \ell)\right) q^{g(g+1) / 4-1 / 2} \ell^{1-g},
\end{aligned}
$$

and thus:

\section{Proposition 2.3.}

$$
\begin{array}{rl}
v_{g} r(q) q^{g(g+1) / 4} \ell^{-1}-2 & c(g, \ell) q^{g(g+1) / 4-1 / 2} \\
& \leq I_{\ell}(q, g) \\
& \leq v_{g} r(q) q^{g(g+1) / 4} \ell^{-1}+\left(v_{g}+3 c(g, \ell)\right) q^{g(g+1) / 4-1 / 2} .
\end{array}
$$

Combining this with our earlier result, we get

$$
\begin{aligned}
\frac{v_{g} r(q) q^{g(g+1) / 4} \ell^{-1}-2 c(g, \ell) q^{g(g+1) / 4-1 / 2}}{v_{g} r(q) q^{g(g+1) / 4}+} & \left.v_{g}+3 c(g, 1)\right) q^{g(g+1) / 4-1 / 2} \\
& \leq \frac{I_{\ell}(q, g)}{I(q, g)} \\
& \leq \frac{v_{g} r(q) q^{g(g+1) / 4} \ell^{-1}+\left(v_{g}+3 c(g, \ell)\right) q^{g(g+1) / 4-1 / 2}}{v_{g} r(q) q^{g(g+1) / 4}-2 c(g, 1) q^{g(g+1) / 4-1 / 2}} .
\end{aligned}
$$

Thus we have:

Theorem 4. For fixed $g$,

$$
\lim _{r \rightarrow \infty} \frac{I_{\ell}\left(p^{r}, g\right)}{I\left(p^{r}, g\right)}=\frac{1}{\ell}
$$

from which Theorem 3 follows immediately.

\section{The general case}

Obviously, an identical argument could be used to establish the fraction of isogeny classes of dimension $g$ for which $P(x) \equiv y$ modulo $\ell$ for any $x$ and $y$ in $\mathbf{Z}$. More generally, we can establish the fraction of isogeny classes of dimension $g$ for which $P(T) \equiv f(T)$ modulo $\ell$ for any given polynomial $f(T)$ of the correct form. Fix

$f(T)=\left(T^{2 g}+q^{g}\right)+m_{1}\left(T^{2 g-1}+q^{g-1} T\right)+\cdots+m_{g-1}\left(T^{g+1}+q T^{g-1}\right)+m_{g} T^{g}$.

For fixed $p$ and $\ell$, let $e_{r, g}$ be the fraction of isogeny classes of $g$-dimensional abelian varieties over $\mathbf{F}_{p^{r}}$ such that $P(T) \equiv f(T)$ modulo $\ell$.

Theorem 5. For fixed $g$,

$$
\lim _{r \rightarrow \infty} e_{r, g}=\frac{1}{\ell^{g}}
$$


Proof. We can follow the same argument as we did for Theorem 3 . Let $J_{\ell}(q, g)=e_{r, g} I(q, g)$ be the number of isogeny classes of $g$-dimensional abelian varieties over $\mathbf{F}_{p^{r}}=\mathbf{F}_{q}$ such that $P(T) \equiv f(T)$ modulo $\ell$. Then our bounds on $J_{\ell}(q, g)=I_{m_{1}, \ldots, m_{g}}(q, g)$ and $I(q, g)$ give us

$$
\begin{aligned}
& \frac{v_{g} r(q) q^{g(g+1) / 4} \ell^{-g}-2 c(g, \ell) q^{g(g+1) / 4-1 / 2} \ell^{1-g}}{v_{g} r(q) q^{g(g+1) / 4}+\left(v_{g}+3 c(g, 1)\right) q^{g(g+1) / 4-1 / 2}} \\
& \leq \frac{J_{\ell}(q, g)}{I(q, g)} \\
& \leq \frac{v_{g} r(q) q^{g(g+1) / 4} \ell^{-g}+\left(v_{g}+3 c(g, \ell)\right) q^{g(g+1) / 4-1 / 2} \ell^{1-g}}{v_{g} r(q) q^{g(g+1) / 4}-2 c(g, 1) q^{g(g+1) / 4-1 / 2}} .
\end{aligned}
$$

On taking the limit, the theorem follows.

\section{References}

[1] Jeffrey D. Achter and Joshua Holden, Notes on an analogue of the Fontaine-Mazur conjecture. J. Théor. Nombres Bordeaux 15 no.3 (2003), 627-637.

[2] Stephen A. DiPipPo and Everett W. Howe, Real polynomials with all roots on the unit circle and abelian varieties over finite fields. J. Number Theory 73 (1998), 426-450.

[3] Gerhard Frey, Ernst Kani and Helmut Völklein, Curves with infinite K-rational geometric fundamental group. In Helmut Völklein, David Harbater, Peter Müller and J. G. Thompson, editors, Aspects of Galois theory (Gainesville, FL, 1996), volume 256 of London Mathematical Society Lecture Note Series, 85-118. Cambridge Univ. Press, 1999.

[4] Joshua Holden, On the Fontaine-Mazur Conjecture for number fields and an analogue for function fields. J. Number Theory 81 (2000), 16-47.

[5] Y. IHARA, On unramified extensions of function fields over finite fields. In Y. IHARA, editor, Galois Groups and Their Representations, volume 2 of Adv. Studies in Pure Math. 89-97. North-Holland, 1983.

Joshua Holden

Department of Mathematics

Rose-Hulman Institute of Technology

Terre Haute, IN 47803, USA

E-mail : holden@rose-hulman.edu 